\title{
ON THE GAUSS MAP OF GENERALIZED SLANT CYLINDRICAL SURFACES
}

\author{
Dong-Soo KIm ${ }^{\mathrm{a}, *}$ AND Booseon Song ${ }^{\mathrm{b}}$
}

\begin{abstract}
In this article, we study the Gauss map of generalized slant cylindrical surfaces (GSCS's) in the 3-dimensional Euclidean space $\mathbb{E}^{3}$. Surfaces of revolution, cylindrical surfaces and tubes along a plane curve are special cases of GSCS's. Our main results state that the only GSCS's with Gauss map $G$ satisfying $\Delta G=A G$ for some $3 \times 3$ matrix $A$ are the planes, the spheres and the circular cylinders.
\end{abstract}

\section{Introduction ANd Preliminaries}

The notion of finite type submanifolds in Euclidean or pseudo-Euclidean space, introduced by B.-Y. Chen during the late 1970's, has become a useful tool for investigating and characterizing many important submanifolds (cf. $[3,4])$. In $[2,6]$ the notion of finite type was extended to differential maps, in particular, to Gauss map of submanifolds.

Let $M$ be a surface of the Euclidean 3-space $\mathbb{E}^{3}$. The map $G: M \rightarrow S^{2} \subset \mathbb{E}^{3}$ which sends each point of $M$ to the unit normal vector to $M$ at the point is called the Gauss map of the surface $M$, where $S^{2}$ is the unit sphere in $\mathbb{E}^{3}$ centered at the origin.

For the matrix $g=\left(g_{i j}\right)$ consisting of the components of the metric on $M$, we denote by $g^{-1}=\left(g^{i j}\right)$ (resp. $\mathcal{G}$ ) the inverse matrix (resp. the determinant) of the matrix $\left(g_{i j}\right)$. The Laplacian $\Delta$ on $M$ is, in turn, given by

$$
\Delta=-\frac{1}{\sqrt{\mathcal{G}}} \sum_{i, j} \frac{\partial}{\partial x^{i}}\left(\sqrt{\mathcal{G}} g^{i j} \frac{\partial}{\partial x^{j}}\right) \text {. }
$$

Received by the editors January 19, 2013. Revised April 26, 2013. Accepted May 21, 2013. 2010 Mathematics Subject Classification. 53A05.

Key words and phrases. Gauss map, Laplace operator, surface of rotation, cylindrical surface, slant cylindrical surface, generalized slant cylindrical surface.

This study was financially supported by Chonnam National University, 2012.

${ }^{*}$ Corresponding author. 
If a submanifold $M$ of Euclidean or pseudo-Euclidean space has 1-type Gauss map $G$, then $G$ satisfies

$$
\Delta G=\lambda(G+C)
$$

for some $\lambda \in \mathbb{R}$ and some constant vector $C$, where $\Delta$ is the Laplace operator corresponding to the induced metric on $M$. Generalizing (1.1), many authors studied various surfaces with Gauss map $G$ satisfying

$$
\Delta G=f(G+C)
$$

for some constant vector $C$ and some smooth function $f([5,8,9,10,11,13])$. Gauss map of a surface satisfying (1.2) is called a pointwise 1-type Gauss map.

On the other hand, Dillen et al. studied surfaces of revolution with Gauss map satisfying

$$
\Delta G=A G
$$

for some $3 \times 3$ matrix $A$, which was inspired by a theorem of Ruh and Vilms on surfaces of constant mean curvature ([14]). As a result, they proved ([7]).

Proposition 1. Among the surfaces of revolution in $\mathbb{E}^{3}$, the only ones whose Gauss map satisfies (1.4) are the planes, the spheres and the circular cylinders.

Baikoussis and Blair also studied ruled surfaces and proved ([1]).

Proposition 2. Among the ruled surfaces in $\mathbb{E}^{3}$, the only ones whose Gauss map satisfies (1.4) are the planes and the circular cylinders.

In [12], the first author with Y.H. Kim introduced the family of generalized slant cylinders (GSCS's). Surfaces of revolution, cylindrical surfaces and tubes along a plane curve are special cases of GSCS's. See Section 2 for the definition and properties of GSCS's.

Here, we give examples of GSCS's with Gauss map satisfying (1.4).

Examples. (1) Plane: $z=0$. In this case, $G=(0,0,1)$ so $\Delta G=0$ and the plane satisfies (1.4) with

$$
A=\left(\begin{array}{lll}
* & * & 0 \\
* & * & 0 \\
* & * & 0
\end{array}\right) .
$$

(2) Cylinder: $(x-a)^{2}+(y-b)^{2}=r^{2}$. In this case, we have $G=\frac{1}{r}(x-a, y-b, 0)$ 
so the cylinder satisfies (1.4) with

$$
A=\left(\begin{array}{ccc}
\frac{1}{r^{2}} & 0 & * \\
0 & \frac{1}{r^{2}} & * \\
0 & 0 & *
\end{array}\right) .
$$

(2) Sphere: $(x-a)^{2}+(y-b)^{2}+(z-c)^{2}=r^{2}$. In this case, we have $G=\frac{1}{r}(x-$ $a, y-b, z-c)$ so the sphere satisfies (1.4) with $A=\frac{2}{r^{2}} I$, where $I$ denotes the identity matrix.

In this paper, we study the GSCS's with Gauss map satisfying (1.4). As a result, we establish

Theorem 3.5. Let $M$ denote a generalized slant cylindrical surface in the 3dimensional Euclidean space $\mathbb{E}^{3}$. Suppose that the Gauss map $G$ of $M$ satisfies $\Delta G=A G$ for some $3 \times 3$ matrix $A$. Then $M$ is part of a plane, a sphere or a circular cylinder.

Hereafter, all objects are assumed to be connected and smooth, unless mentioned otherwise.

\section{Generalized Slant Cylindrical Surfaces}

For a fixed unit speed plane curve $\alpha(s)=(x(s), y(s), 0)$, let $T(s)=\alpha^{\prime}(s)$ and $N(s)=\left(-y^{\prime}(s), x^{\prime}(s), 0\right)$ denote the unit tangent and principal normal vector, respectively. The curvature $\kappa(s)$ of $\alpha(s)$ is defined by $T^{\prime}(s)=\kappa(s) N(s)$ and we have $T(s) \times N(s)=V$, where $V$ denotes the unit vector $(0,0,1)$. For a constant $\theta$, we let $\beta_{s}(t)=t(\cos \theta N(s)+\sin \theta V)$. Then the ruled surface $M$ defined by

$$
X(s, t)=\alpha(s)+\beta_{s}(t)
$$

is regular at $(s, t)$ where $1-\cos \theta \kappa(s) t$ does not vanish. This ruled surface $M$ is called a slant cylindrical surface (SCS) over $\alpha(s)$. The SCS with $\sin \theta=0$ or $\cos \theta=0$ is nothing but a parametrization of either a plane or a cylindrical surface.

In general, we consider another unit speed plane curve $\beta(t)=(z(t), w(t))$. If we let $\beta_{s}(t)=z(t) N(s)+w(t) V$, then the parametrized surface defined by

$$
X(s, t)=\alpha(s)+\beta_{s}(t)
$$

is regular at $(s, t)$ where $1-\kappa(s) z(t)$ does not vanish. This parametrized surface $M$ is called a generalized slant cylindrical surface (GSCS) over $\alpha(s)$. 
If $\beta(t)$ is a straight line (resp., a circle), then the GSCS $X(s, t)$ is nothing but an SCS (resp., a tube) along a plane curve $\alpha$. If $\alpha(s)$ is a straight line, then the GSCS $X(s, t)$ is a cylindrical surface over a plane curve. Furthermore, we have the following ([12]).

Proposition 2.1. If $\alpha(s)$ is a circle, then a GSCS $M$ over $\alpha(s)$ is a surface of revolution.

Therefore we see that cylindrical surfaces, tubes along a plane curve and surfaces of revolution are special cases of GSCS's.

We also have the following characterizations ([10]):

Proposition 2.2. Let $M$ denote a GSCS given by (2.2). Then we have the following.

(1) If the mean curvature $H$ is constant, then $M$ is a surface of revolution.

(2) If the Gaussian curvature $K$ is constant, then $M$ is either a surface of revolution or an $S C S$.

\section{Gauss Map of GSCS'S}

Let $\alpha(s)=(x(s), y(s), 0)$ be a unit speed plane curve with the Frenet frame $T(s)$ and $N(s)$ which is defined on an interval $I$. We consider a GSCS $M$ parametrized by

$$
X(s, t)=\alpha(s)+\beta_{s}(t), \quad(s, t) \in I \times J,
$$

where $\beta(t)=(z(t), w(t))$ is a unit speed plane curve, $\beta_{s}(t)=z(t) N(s)+w(t) V$, and $V=(0,0,1)$. Without loss of generality, we may assume that $\beta(0)=0$, hence we have $X(s, 0)=\alpha(s)$ for all $s \in I$.

Then $X(s, t)$ is regular at $(s, t)$ where $q(s, t)=1-\kappa(s) z(t)$ does not vanish and we get

$$
\begin{aligned}
& X_{s}=q(s, t) T(s), X_{t}=z^{\prime}(t) N(s)+w^{\prime}(t) V, \\
& G(s, t)=-w^{\prime}(t) N(s)+z^{\prime}(t) V .
\end{aligned}
$$

The Laplacian $\Delta$ on $M$ is given by for $f \in C^{\infty}(M)$

$$
\Delta f=-q^{-3}\left\{\kappa^{\prime}(s) z(t) f_{s}+q f_{s s}-q^{2} \kappa(s) z^{\prime}(t) f_{t}+q^{3} f_{t t}\right\} .
$$

Hence it follows from (3.2) and (3.3) that

$$
\begin{aligned}
-q^{3} \Delta G= & \kappa^{\prime}(s) w^{\prime}(t) T(s)+q\left\{\kappa(s)^{2} w^{\prime}(t)+q \kappa(s) z^{\prime}(t) w^{\prime \prime}(t)\right. \\
& \left.-q^{2} w^{\prime \prime \prime}(t)\right\} N(s)+q^{2}\left\{-\kappa(s) z^{\prime}(t) z^{\prime \prime}(t)+q z^{\prime \prime \prime}(t)\right\} V .
\end{aligned}
$$


First, we show that a slant cylindrical surface with Gauss map $G$ satisfying (1.4) is an open part of either a plane or a circular cylinder. Since an SCS is a ruled surface, Proposition 3.1 can be deduced from the results in [1]. But, for conveniences, we give a proof.

Proposition 3.1. Let $M$ be an SCS given by (3.1) with $z(t)=t \cos \theta$ and $w(t)=$ $t \sin \theta$. Suppose that the Gauss map $G$ of $M$ satisfies $\Delta G=A G$ for some $3 \times 3$ matrix $A$. Then $M$ is an open part of either a plane or a circular cylinder.

Proof. Since $z(t)=t \cos \theta$ and $w(t)=t \sin \theta$, it follows from (3.2) and (3.4) that

$$
G(s, t)=-\sin \theta N(s)+\cos \theta V=\left(\sin \theta y^{\prime}(s),-\sin \theta x^{\prime}(s), \cos \theta\right)
$$

and

$$
-q^{3} \Delta G=\kappa^{\prime}(s) \sin \theta T(s)+q \kappa(s)^{2} \sin \theta N(s) .
$$

By the assumption, we obtain

$$
\begin{aligned}
& \sin \theta\left\{x^{\prime} \kappa^{\prime}-y^{\prime} \kappa^{2} q\right\}=-q^{3}\left\{\left(a_{11} y^{\prime}-a_{12} x^{\prime}\right) \sin \theta+a_{13} \cos \theta\right\} \\
& \sin \theta\left\{y^{\prime} \kappa^{\prime}+x^{\prime} \kappa^{2} q\right\}=-q^{3}\left\{\left(a_{21} y^{\prime}-a_{22} x^{\prime}\right) \sin \theta+a_{23} \cos \theta\right\}
\end{aligned}
$$

and

$$
a_{31} \sin \theta y^{\prime}(s)-a_{32} \sin \theta x^{\prime}(s)+a_{33} \cos \theta=0 .
$$

It follows from $x^{\prime}(s) \times(3.7)+y^{\prime}(s) \times(3.8)$ and $x^{\prime}(s) \times(3.8)-y^{\prime}(s) \times(3.7)$ that

$$
\begin{aligned}
& \sin \theta \kappa^{\prime}(s)=q^{3} f(s)=(1-t \cos \theta \kappa(s))^{3} f(s), \\
& \sin \theta \kappa(s)^{2}=q^{2} g(s)=(1-t \cos \theta \kappa(s))^{2} g(s),
\end{aligned}
$$

where $f(s)$ and $g(s)$ are given by

$$
\begin{aligned}
& f(s)=\sin \theta\left\{a_{12}\left(x^{\prime}\right)^{2}+\left(a_{22}-a_{11}\right) x^{\prime} y^{\prime}-a_{21}\left(y^{\prime}\right)^{2}\right\}-\cos \theta\left\{a_{13} x^{\prime}+a_{23} y^{\prime}\right\}, \\
& g(s)=\sin \theta\left\{a_{22}\left(x^{\prime}\right)^{2}-\left(a_{12}+a_{21}\right) x^{\prime} y^{\prime}+a_{11}\left(y^{\prime}\right)^{2}\right\}-\cos \theta\left\{a_{23} x^{\prime}-a_{13} y^{\prime}\right\} .
\end{aligned}
$$

We divide by two cases as follows.

Case 1. $\cos \theta \neq 0$.

If $\sin \theta=0$ or $\kappa(s)$ vanishes identically, then $M$ is a part of a plane. Hence we may suppose that $\sin \theta \neq 0$ and $\kappa(s) \neq 0$ on an interval $I_{0}$. Then, the second equation in (3.10) shows that $t$ is a function of $s \in I_{0}$, which is a contradiction. Case 2. $\cos \theta=0$. 
In this case, $M$ is a cylindrical surface over $\alpha(s)$. We may assume that $\sin \theta=1$. Hence, from (3.10) and (3.11) we get

$$
\kappa^{\prime}(s)=a_{12} x^{\prime}(s)^{2}+\left(a_{22}-a_{11}\right) x^{\prime}(s) y^{\prime}(s)-a_{21} y^{\prime}(s)^{2}
$$

and

$$
\kappa(s)^{2}=a_{22} x^{\prime}(s)^{2}-\left(a_{12}+a_{21}\right) x^{\prime}(s) y^{\prime}(s)+a_{11} y^{\prime}(s)^{2} .
$$

By differentiating (3.13) and using $y^{\prime} y^{\prime \prime}=-x^{\prime} x^{\prime \prime}$, we obtain

$$
2 \kappa^{\prime} \kappa=2\left(a_{22}-a_{11}\right) x^{\prime} x^{\prime \prime}-\left(a_{12}+a_{21}\right)\left\{x^{\prime \prime} y^{\prime}+x^{\prime} y^{\prime \prime}\right\} .
$$

Since $\kappa(s) y^{\prime}(s)=-x^{\prime \prime}(s)$, multiplying the both sides of (3.14) by $y^{\prime}(s)$ and then using (3.12), we get

$$
x^{\prime \prime}(s)\left\{4 \kappa^{\prime}(s)+\left(a_{21}-a_{12}\right)\right\}=0 .
$$

Suppose that $I_{0}=\left\{s \in I \mid x^{\prime \prime}(s) \neq 0\right\}$ is nonempty. Then on $I_{0}, \kappa^{\prime}(s)$ is constant. Hence, by differentiating (3.12) and then multiplying $y^{\prime}$, on $I_{0}$ we obtain

$$
\left(a_{22}-a_{11}\right)\left\{y^{\prime}(s)^{2}-x^{\prime}(s)^{2}\right\}+2\left(a_{12}+a_{21}\right) x^{\prime}(s) y^{\prime}(s)=0 .
$$

Hence, it follows from (3.13) that on $I_{0}$

$$
2 \kappa(s)^{2}=a_{11}+a_{22},
$$

which is a nonzero constant because $\kappa(s) y^{\prime}(s)=-x^{\prime \prime}(s) \neq 0$ on the interval $I_{0}$.

If the complement $I_{0}^{c}$ of $I_{0}$ has nonempty interior, then $\kappa(s)=0$ there. Thus, by the continuity of $\kappa(s)$ we see that $\kappa(s)$ is constant on the whole domain $I$ of $\alpha$. Therefore, $M$ is an open portion of either a plane or a circular cylinder.

Combining Cases 1 and 2 completes the proof of Proposition 3.1.

Now, suppose that the Gauss map $G$ of a GSCS $M$ defined by (3.1) satisfies (1.4) for some $3 \times 3$ matrix $A$. Then, from (3.2) and (3.4) we get

$$
\begin{aligned}
& x^{\prime} \kappa^{\prime} w^{\prime}-p q y^{\prime}=-q^{3}\left\{a_{11} y^{\prime} w^{\prime}-a_{12} x^{\prime} w^{\prime}+a_{13} z^{\prime}\right\}, \\
& y^{\prime} \kappa^{\prime} w^{\prime}+p q x^{\prime}=-q^{3}\left\{a_{21} y^{\prime} w^{\prime}-a_{22} x^{\prime} w^{\prime}+a_{23} z^{\prime}\right\},
\end{aligned}
$$

and

$$
-\kappa z^{\prime} z^{\prime \prime}+q z^{\prime \prime \prime}=-q\left\{\left(a_{31} y^{\prime}-a_{32} x^{\prime}\right) w^{\prime}+a_{33} z^{\prime}\right\}
$$

where

$$
p(s, t)=\kappa(s)^{2} w^{\prime}(t)+q \kappa(s) z^{\prime}(t) w^{\prime \prime}(t)-q^{2} w^{\prime \prime \prime}(t) .
$$


It follows from $x^{\prime}(s) \times(3.18)+y^{\prime}(s) \times(3.19)$ and $x^{\prime}(s) \times(3.19)-y^{\prime}(s) \times(3.18)$ that

$$
\kappa^{\prime} w^{\prime}=-q^{3}\left[\left\{\left(a_{11}-a_{22}\right) x^{\prime} y^{\prime}-a_{12}\left(x^{\prime}\right)^{2}-a_{21}\left(y^{\prime}\right)^{2}\right\} w^{\prime}+\left(a_{13} x^{\prime}+a_{23} y^{\prime}\right) z^{\prime}\right]
$$

and

$$
p=q^{2}\left[\left\{\left(a_{11}\left(y^{\prime}\right)^{2}-\left(a_{12}+a_{21}\right) x^{\prime} y^{\prime}+a_{22}\left(x^{\prime}\right)^{2}\right\} w^{\prime}+\left(a_{13} y^{\prime}-a_{23} x^{\prime}\right) z^{\prime}\right] .\right.
$$

First, we prove

Lemma 3.2. Let $M$ be a GSCS given by (3.1). Suppose that the Gauss map $G$ of $M$ satisfies $\Delta G=A G$ for some $3 \times 3$ matrix $A$ with $a_{13}=a_{23}=0$. Then $M$ is open part of either an SCS or a surface of revolution.

Proof. It follows from (3.22) that

$$
w^{\prime}(t)\left\{\kappa^{\prime}(s)-q^{3} f(s)\right\}=0,
$$

where $f(s)$ is a function of $s$.

Let's denote by $J_{0}=\left\{t \in J \mid w^{\prime}(t) \neq 0\right\}$. If $J_{0}$ is empty, then $M$ is part of a plane parallel to the $x y$-plane. Otherwise, we have $\kappa^{\prime}(s)=q^{3} f(s)$ for all $s \in I$. Recall that $q=1-\kappa(s) z(t)$.

Suppose that $I_{0}=\left\{s \in I \mid \kappa^{\prime}(s) \neq 0\right\}$ is nonempty. Then, it follows from (3.24) that on $I_{0} \times J_{0}$ we obtain

$$
\kappa(s) z(t)=1-\left(\frac{\kappa^{\prime}(s)}{f(s)}\right)^{1 / 3}
$$

which shows that $z(t)$ is constant on $J_{0}$. This shows that $J_{0}$ is the whole domain $J$ of $\beta$ and hence $\beta$ is a straight line perpendicular to the $x y$-plane. Thus on $I_{0} \times J$, $M$ is a cylindrical surface over $\alpha$. Due to Proposition 3.1, we see that on $I_{0}, \alpha$ has constant curvature $\kappa$, which is a contradiction. Therefore $\kappa(s)$ is constant on the whole domain $I$.

The above discussion implies that when $w^{\prime}(t) \neq 0$ for some $t, \alpha$ is either a straight line or a circle. If $\alpha$ is a straight line, then $M$ is a cylindrical surface over a plane curve. That is, $M$ is an SCS. If $\alpha$ is a circle, then $M$ is a surface of revolution.

This completes the proof of Lemma 3.2.

Next, we show

Lemma 3.3. Let $M$ be a GSCS given by (3.1). Suppose that the Gauss map $G$ of $M$ satisfies $\Delta G=A G$ for some $3 \times 3$ matrix $A$ with $a_{31}=a_{32}=0$. Then $M$ is an open part of an SCS or a surface of revolution. 
Proof. It follows from (3.20) that

$$
\kappa(s)\left\{z(t)\left(z^{\prime \prime \prime}(t)+a_{33} z^{\prime}(t)\right)+z^{\prime}(t) z^{\prime \prime}(t)\right\}=z^{\prime \prime \prime}(t)+a_{33} z^{\prime}(t) .
$$

If $z^{\prime \prime \prime}(t)+a_{33} z^{\prime}(t)$ is nonzero for some $t$, then $\kappa(s)$ is a nonzero constant. Hence $M$ is a surface of revolution.

Otherwise, that is, $z^{\prime \prime \prime}(t)+a_{33} z^{\prime}(t)$ vanishes identically, then (3.26) implies that

$$
\kappa(s) z^{\prime}(t) z^{\prime \prime}(t)=0 .
$$

This shows that $\kappa(s)=0$ or $z^{\prime}(t)$ is constant, that is, $\alpha$ or $\beta$ is a straight line. Hence $M$ is a slant cylindrical surface.

This completes the proof of Lemma 3.3.

Finally, we prove the following.

Lemma 3.4. Let $M$ be a GSCS given by (3.1). Suppose that the Gauss map $G$ of $M$ satisfies $\Delta G=A G$ for some arbitrary $3 \times 3$ matrix $A$. Then $M$ is an open part of either an SCS or a surface of revolution.

Proof. From (3.20), we get

$$
\kappa(s) z^{\prime}(t) z^{\prime \prime}(t)-q z^{\prime \prime \prime}(t)=q\left\{f(s) w^{\prime}(t)+a_{33} z^{\prime}(t)\right\},
$$

where

$$
f(s)=a_{31} y^{\prime}(s)-a_{32} x^{\prime}(s) .
$$

Note that $\beta(0)=(z(0), w(0))=0$. We divide by two cases.

Case 1. Suppose that $w^{\prime}(0) \neq 0$. In this case, since $q(0)=1$, putting $t=0$ in (3.28), we obtain

$$
f(s) w^{\prime}(0)=\kappa(s) z^{\prime}(0) z^{\prime \prime}(0)-\left(z^{\prime \prime \prime}(0)+a_{33} z^{\prime}(0)\right) .
$$

This shows that

$$
f(s)=a \kappa(s)+b
$$

for some constants $a$ and $b$ given by

$$
a=z^{\prime}(0) z^{\prime \prime}(0) / w^{\prime}(0), b=-\left(z^{\prime \prime \prime}(0)+a_{33} z^{\prime}(0)\right) / w^{\prime}(0) .
$$

If $a=0$, then as in the proof of Lemma 3.3, we may prove that $M$ is an open part of either an SCS or a surface of revolution. Hence we may assume that $a$ is nonzero. 
Since $q=1-\kappa(s) z(t)$, substituting $f(s)$ in (3.31) into (3.28), we see that the curvature function $\kappa(s)$ of $\alpha$ satisfies the following quadratic polynomial:

$$
g(t) \kappa(s)^{2}+h(t) \kappa(s)+k(t)=0,
$$

where

$$
\begin{aligned}
& g(t)=a z(t) w^{\prime}(t), \quad k(t)=-z^{\prime \prime \prime}(t)-a_{33} z^{\prime}(t), \\
& h(t)=b z(t) w^{\prime}(t)+z^{\prime}(t) z^{\prime \prime}(t)-a w^{\prime}(t)-z(t) k(t) .
\end{aligned}
$$

Therefore, it follows from (3.33) that $\kappa(s)$ is constant, unless the coefficients $g(t), h(t)$ and $k(t)$ identically vanish. In this case, $M$ is either a surface of revolution or a cylindrical surface.

If the coefficients $g(t), h(t)$ and $k(t)$ identically vanish, then it follows from (3.34) that $z(t) z^{\prime}(t) z^{\prime \prime}(t)$ vanishes identically. This shows that $\beta(t)$ is a straight line. Hence, we see that $M$ is an SCS.

Case 2. Suppose that $w^{\prime}(0)=0$. In this case, we may assume that $\beta$ is not a straight line because otherwise, $M$ is an SCS. Then, for some $t_{0}$, we have $w^{\prime}\left(t_{0}\right) \neq 0$.

Putting $\bar{z}(t)=z(t)-c$ with $c=z\left(t_{0}\right)$, we consider the parametrization $\bar{X}$ of $M$ given by

$$
\bar{X}(s, t)=\bar{\alpha}(s)+\bar{z}(t) N(s)+w(t) V,
$$

where the base curve $\bar{\alpha}$ is a parallel curve of $\alpha$ defined by $\bar{\alpha}(s)=\alpha(s)+c N(s)$.

For an arc length parameter $u$ of $\bar{\alpha}, M$ has the reparametrization $\bar{X}(u, t)=$ $\bar{\alpha}(u)+\bar{z}(t) N(u)+w(t) V$ of $X(s, t)$ with $\bar{z}\left(t_{0}\right)=0$ and $w^{\prime}\left(t_{0}\right) \neq 0$. Hence, we can proceed as in the proof of Case 1 to show that $M$ is an open part of either a surface of revolution or an SCS. This completes the proof of Case 2 .

Combining Cases 1 and 2 completes the proof of Lemma 3.4.

Now, we combine Lemma 3.4, Proposition 3.1 and Proposition 1 in Section 1. Then, we get the following theorem.

Theorem 3.5. Let $M$ denote a generalized slant cylindrical surface in the 3dimensional Euclidean space $\mathbb{E}^{3}$. Suppose that the Gauss map $G$ of $M$ satisfies $\Delta G=A G$ for some $3 \times 3$ matrix $A$. Then $M$ is an open part of a plane, a sphere or a circular cylinder. 


\section{REFERENCES}

1. C. Baikoussis \& D.E. Blair: On the Gauss map of ruled surfaces. Glasgow Math. J. 34 (1992), 355-359.

2. C. Baikoussis, B.-Y. Chen \& L. Verstraelen: Ruled surfaces and tubes with finite type Gauss map. Tokyo J. Math. 16 (1993), 341-348.

3. B.-Y. Chen: Total mean curvature and submanifolds of finite type. World Scientific Publ., New Jersey, 1984.

4. B.-Y. Chen: Finite type submanifolds and generalizations. University of Rome, 1985.

5. B.-Y. Chen, M. Choi \& Y.H. Kim: Surfaces of revolution with pointwise 1-type Gauss map. J. Korean Math. Soc. 42 (2005), 447-455.

6. B.-Y. Chen \& P. Piccinni: Submanifolds with finite type Gauss map. Bull. Austral. Math. Soc. 35 (1987), 161-186.

7. F. Dillen, J. Pas \& L. Verstraelen: On the Gauss map of surfaces of revolution. Bull. Inst. Math. Acad. Sinica 18 (1990), no. 3, 239-246.

8. U. Dursun: Hypersurfaces with pointwise 1-type Gauss map. Taiwanese J. Math. 11 (2007), no. 5, 1407-1416.

9. __ Flat surfaces in the Euclidean space $E^{3}$ with pointwise 1-type Gauss map. Bull. Malays. Math. Sci. Soc.(2) 33 (2010), no. 3, 469-478.

10. D.-S. Kim: Surfaces with pointwise 1-type Gauss map. J. Korean Soc. Math. Educ. Ser. B Pure Appl. Math. 18 (2011), no. 4, 369-377.

11. _ : Surfaces with pointwise 1-type Gauss map of the second kind. J. Korea Soc. Math. Educ. Ser. B: Pure Appl. Math. 19 (2012), no. 3, 229-237.

12. D.-S. Kim \& Y.H. Kim: Surfaces with planar lines of curvature. Honam Math. J. 32 (2010), 777-790.

13. Y.H. Kim \& D.W. Yoon: On the Gauss map of ruled surfaces in Minkowski space. Rocky Mountain J. Math. 35 (2005), no. 5, 1555-1581.

14. E.A. Ruh \& J. Vilms: The tension field of the Gauss map. Trans. Amer. Math. Soc. 149 (1970), 569-573.

a Department of Mathematics, Chonnam National University, Kwanguu 500-757, Korea Email address: dosokim@chonnam.ac.kr

b Department of Mathematics, Chonnam National University, Kwanguu 500-757, Korea

Email address: booseons@gmail.com 